\title{
Ein Tag Coaching: Evaluation der Wirksamkeit von Coachings nach dem Modell der Coachingkonzeption „Systemisch-konstruktivistisches Einzel- und Teamcoaching im Management" SKETM
}

\author{
Hansjörg Künzli'
}

Online publiziert: 15 . September 2016

(C) Der/die Autor(en) 2016. Dieser Artikel ist eine Open-Access-Publikation.

Zusammenfassung Anhand einer explorativen Fragestellung wurde der Einfluss von Coaching, basierend auf dem Modell „Systemisch-konstruktivistisches Einzel- und Teamcoaching im Management" SKETM auf verschiedene Ergebnisindikatoren überprüft. Wichtige Merkmale von Coachings auf der Basis des Modells SKETM sind Prozessdirektivität und -transparenz bei gleichzeitiger inhaltlicher Abstinenz. SKETM-Coachings werden innerhalb eines Tages durchgeführt.

Zur Überprüfung der Fragestellung wurde ein quasiexperimentelles Wartekontrollgruppendesign mit drei Messzeitpunkten verwendet. Von insgesamt 64 Coachees zum Zeitpunkt t0 konnten 33 der Wartekontrollgruppe und 31 der Interventionsgruppe zugewiesen werden. Am Versuch beteiligten sich 25 Coaches, die nach der Methode SKETM arbeiten. Als Ergebnisindikatoren wurden eine Zielerreichungsskala, der Fragebogen Evaluation Coaching - Klärung und Umsetzung des Anliegens FEC-KU und Teile des Inkongruenzfragebogens INK verwendet. Interaktions- und Zeiteffekte wurden mit der Prozedur Generalized Estimating Equation GEE ausgewertet.

Die Auswertungen zeigten signifikante Zeiteffekte $(\mathrm{t} 0-\mathrm{t} 2)$ und kleine bis hohe Effekte für alle Skalen (Zielannäherung $d=1,51$; Inkongruenzskalen $d=0,44-0,94$; Anliegenklärung und -umsetzung $d=1,02-1,44)$. Mit Ausnahme von drei Subskalen des INK wurden alle Interaktionen (t0-t1) signifikant. Die Effekte bewegten sich im tiefen bis hohen Bereich (Zielannäherung $d=1,52$;

Hansjörg Künzli

kasg@ zhaw.ch

1 Department of Applied Psychology, Zurich University of Applied Sciences (ZHAW), Pfingstweidstrasse 96, 8037 Zürich, Schweiz
Inkongruenzskalen $d=0,34-0,88$; Anliegenklärung und Umsetzung $d=0,89-1,44)$. Vom zweiten auf den dritten Messzeitpunkt blieben die Werte der Interventionsgruppe auf den Skalen der Anliegenklärung und -umsetzung stabil, die Werte der Inkongruenzskalen verbesserten sich signifikant.

Coaching nach dem Modell „SKETM“ ist eine wirksame Intervention, die bei unterschiedlichen Anliegen zur Zielannäherung, der Anliegenklärung und -umsetzung sowie der Reduzierung des Inkongruenzerlebens beiträgt. Die gemessenen Effekte sind im Vergleich zu den Werten aus den bisher bekannten Metaanalysen zur Wirksamkeit von Coaching gleich hoch oder höher.

Erste quasiexperimentelle Feldstudie zu systemisch-konstruktivistischem Coaching.

Schlüsselwörter Systemisch-konstruktivistisches Coaching · Inkongruenz · Anliegenklärung ·

Zielannäherung

\section{One Day of Coaching: Effectiveness of a Systemic- Constructivist Coaching Approach}

Abstract Using an exploratory approach, the influence of coaching (based on the SKETM model) on a number of results indicators was examined. SKETM stands for ,systemisch-konstruktivistisches Einzel- und Teamcoaching im Management", i.e. systemic-constructivist individual and team coaching in management. The main features of coaching based on the SKETM model are the coach guiding the process, making everything transparent to the client, while concurrently refraining from interfering/mingling with the content. Coachings based on the SKETM method last for one day. 
The study employed a quasi-experimental wait-list design with measures at time 0 , time 1 and time 2 . Out of a total of 64 coachees at time 0 , a number of 33 were assigned to the wait-list control group and 31 to the intervention group. 25 coaches, all working with the SKETM method, participated in the study. The following results indicators were used: a goal attainment scale, a coaching evaluation questionnaire - (Fragebogen Evaluation Coaching - Klärung und Umsetzung des Anliegens FEC-KU); and parts of the incongruence questionnaire INK. Interaction and time effects were evaluated using generalized estimating equation (GEE).

Significant time effects ( $\mathrm{t} 0-\mathrm{t} 2)$ and small to large effects for all scales (goal attainment $d=1.51$; incongruence scales $d=0.44-0.94$; problem clarification and solving $d=$ 1.02-1.44) were observed. With the exception of three INK subscales, all interactions ( $\mathrm{t} 0-\mathrm{t} 1)$ were significant. The effects ranged from low to high (goal attainment $d=1.52$; incongruence scales $d=0.34-0.88$; problem clarification and solving $d=0.89-1.44)$. From the second to the third time of measurement, the values of the intervention group on the problem clarification and solving scales remained constant, the values of the incongruence scales improved significantly.

Coaching based on the SKETM model is an effective intervention method, contributing to goal attainment, problem clarification and solving as well reducing the incongruence perception in a number of issues. The measured effects are equal or higher in comparison to the values obtained in previous meta-analyses on the effectiveness of coaching.

First quasi-experimental field study on systemic-constructivist coaching.

Keywords Systemic-constructivist coaching • Incongruence $\cdot$ Problem clarification - Goal attainment

\section{Einleitung}

Das Angebot und die Inanspruchnahme von Coaching haben in den letzten Jahren zugenommen (Stephan et al. 2010). Parallel dazu ist die Zahl der Wirksamkeitsstudien im Laufe der letzten 20 Jahre kontinuierlich gestiegen (Böning und Kegel 2015; Kotte et al. 2016; Künzli 2009; Möller und Kotte 2011). Trotzdem bleibt ihre Anzahl noch überschaubar. Böning und Kegel (2015) fanden insgesamt 145 empirische Arbeiten zum Thema, davon 61 aus dem Bereich Business-Coaching. Nach wie vor zeichnet sich das Feld der Wirksamkeitsforschung durch große Heterogenität in Hinsicht auf die Definition von Coaching, die theoretische Verankerung, die verwendeten Forschungsdesigns, die Ergebnisindikatoren, die methodische Stringenz aus (Künzli 2009). Experimentelle Studien sind immer noch die Ausnahme (Böning und Kegel 2015; Künzli 2009). Die in den drei verfügbaren Metaanalysen berichteten mittleren gewichteten Effektstärken waren gering ( $g=0,10$; Sonesh et al. 2015), gering bis mittel ( $g=0,36$; Jones et al. 2015) und mittel bis groß ( $g=0,66$; Theeboom et al. 2014). Bei aller Unterschiedlichkeit der in den Metaanalysen verwendeten Ein- und Einschlusskriterien und der Vielfalt der in den verarbeiteten Studien eingesetzten Ergebnisindikatoren scheint sich jedoch abzuzeichnen, dass Interventionen, die unter dem Sammelbegriff Coaching eingeordnet werden, zumindest moderate Wirkungen zeigen. Die Generalisierung dieser Befunde auf jegliche Formen von Coaching ist hingegen nicht zulässig, da der Prozess und die Ergebnisse von Coaching immer Unikate oder Ko-Kreationen (Jaenicke 2009) sind, die aus der Interaktion von Coachee, Coach und weiteren Beteiligten entwickelt werden. So gesehen müsste der Wirksamkeitsnachweis für jeden Einzelfall erbracht werden. Dies wäre aufwändig, aber wie die Single- $n$-design Forschung (Barlow et al. 2009) und die individuumsorientierte Forschung (Künzli \& Stulz 2011) zeigen, mit gewissen methodischen Abstrichen in Bezug auf den Nachweis der kausalen Verbindung zwischen Coaching und Ergebnis durchaus möglich.

Eine wichtige vermittelnde Größe zwischen Individuumsorientierung und einer Betrachtung, die Coaching als Gattung sieht, ist die Ausbildung der Coaches. Ausbildungen vermitteln zukünftigen Coaches grundlegende Menschenbilder, erkenntnistheoretische Setzungen, methodische Anweisungen und Praxeologien bis hin zu Hinweisen für konkretes Handeln (Schreyögg 2009). Zudem gilt die Aussage von Petzold et al. (2003), dass von einer qualitätsvollen Supervisionsausbildung erst dann gesprochen werden kann, wenn die Ausgebildeten das Gelernte erfolgreich in die Praxis transformieren, auch für Coaching. Jede Coaching-Ausbildung muss sich die Frage stellen, ob die Coachings, die im Feld nach ihren Vorgaben durchgeführt werden, auch die gewünschten Wirkungen erzielen. Noch deutlicher formulieren es McKenna und Davis (2009): Coaches, die keine überzeugenden Nachweis ihrer Wirksamkeit erbringen können, werden nach Hause geschickt. Diese Feststellung geht einher mit der Forderung von Lai und MacDowall (2014) nach mehr Kontrollgruppenstudien zur Evaluation einzelner Coaching-Schulen oder Ansätzen. Hintergrund der Forderung ist die Frage nach funktionierenden Coaching-Ansätzen.

Studien zu Coachings, die auf der Basis einer einheitlichen Schule oder Theorie beruhen, liegen vor, sind aber noch selten. $\mathrm{Zu}$ nennen sind hier z. B. die Arbeiten von Green et al. (2006, 2007); Grant et al. (2009) sowie von Grant et al. (2010). Die Coachings in den genannten Studien basierten alle auf einem lösungsorientierten, kognitivbehavioralen Ansatz, der in Grant und Greene (2001) und Greene und Grant (2003) beschrieben ist. Eine Ausnahme 
dazu bilden die Arbeiten von Bozer und Sarros (2012) und Cerni et al. (2010). Die dort untersuchten Coachings basierten auf einem kognitiv-behavioralen Ansatz resp. der Cognitive-Experiential Self Theory CEST von Epstein (1996). Die bisher einzige bekannte Kontrollgruppenstudie, in der ein systemischer Ansatz eine Rolle spielte, ist diejenige von Behrendt (2004). Er untersuchte, ob sich Coachings auf der Basis von Psychodrama von einem Vergleichscoaching auf der Basis von systemischen und Gesprächsführungskonzepten nach Rogers und einer Kontrollgruppe ohne Coaching unterschieden. Studien zu Coachings, die auf einem einheitlich systemischen oder systemisch-konstruktivistischen Hintergrund basieren, existieren bisher nicht. Dies obwohl systemische Ansätze im deutschen Sprachraum nach Aussagen von Coaches häufig verwendet werden (Seiger und Künzli 2012). Folgt man einschlägigen Lehrbüchern zu systemischer Beratung (vgl. z. B. König und Volmer 2012; Loebbert 2013; Radatz 2003; Schmidt 2013; Schlippe und Schweizer 2009) sind diese oft mit einem konstruktivistischen Hintergrund verbunden oder machen Anleihen im Konstruktivismus. Eine empirische Überprüfung von Coachings, die auf systemisch-konstruktivistischen Grundsätzen abgeleitet sind, steht unseres Wissens aber noch aus. Umso mehr erscheint die Evaluation eines Ansatzes, wie er durch das SKETM verkörpert wird, als eine Forschungslücke.

\section{Ergebnisindikatoren von Coachings mit offenem Anliegen}

Auch wenn auf dem Markt immer mehr spezifische Coachingvarianten für unterschiedliche Anliegen angeboten werden (Seiger und Künzli 2012), ist Coaching nach wie vor eine Intervention, die auf die Veränderung vielfältiger Anliegen abzielt (Greif 2008). Heterogenität der Anliegen ist eher die Regel (vgl. z. B. Böning und Fritschle 2005; Lippmann 2013; Rauen 2008) als die Ausnahme. Für eine Feldstudie erschien es daher nicht sinnvoll, diese Heterogenität einzuschränken. Entsprechend wurde Wert darauf gelegt, soweit als möglich sog. allgemein anwendbaren Ergebnisindikatoren (Greif 2008) zu verwenden. Diese sind weitgehend unabhängig vom Inhalt des bearbeiteten Anliegens und sollten sich durch jedes Coaching verändern. Weitgehend durchgesetzt hat sich hier die Zielerreichung (Grant et al. 2009, 2010; Green et al. 2006; Greif 2008). Neben der Zielerreichung nennt Greif (2008) noch weitere allgemein anwendbare Ergebnisindikatoren, z. B. die Verbesserung des Affekts (z. B. Finn et al. 2006; Green et al. 2006; Offermanns 2003) oder das allgemeine Wohlbefinden (z. B. Green et al. 2006; Offermanns 2003; Spence und Grant 2007; Steinmetz 2005). Ein Nachteil dieser Indikatoren liegt jedoch darin, dass sich ihre Bewertung aus der gesamten Lebenssituation und nicht nur aus dem unmittelbar im Coaching bearbeiteten Anliegen speist. In der vorliegenden Studie wurde daher ein anderer Weg beschritten. Um diesem Nachteil zumindest ansatzweise entgegenzuwirken, wurde in dieser Studie der Versuch gewagt, ein neues, noch ungeprüftes Instrument einzusetzen, das nur auf den wahrgenommenen Handlungsfortschritt hinsichtlich des Anliegens Bezug nimmt und damit unabhängig ist vom konkreten Inhalt des Anliegens.

\section{Variablen des Handlungsfortschritts: Klärung und Umsetzung als Ergebnisindikatoren}

Gemeinsamer Nenner hinter der Inanspruchnahme von Coaching ist praktisch immer der Wunsch nach einer verbesserten Handlungs- oder Selbststeuerungsfähigkeit (vgl. z. B. Lippmann 2013; Rauen 2008). Dieser Wunsch kann aus einer Defizitsituation aber auch antizipativ, in Hinsicht auf zukünftige, herausfordernde Situationen entstanden sein (Schreyögg 2003). Der Wunsch bezieht sich aber in der Regel nicht auf eine umfassende Verbesserung der Selbststeuerungsfähigkeit, sondern viel mehr auf ein mehr oder weniger eng begrenztes konkretes Thema, in dem der Coachee eine Veränderung erzielen möchte. Dieses wird im Coaching zunächst oft mit dem wertneutralen Begriff „Anliegen“ umschrieben (vgl. z. B. Lippmann 2013; Schreyögg 2003). Ziel des Coachings ist es dann, das vom Coachee eingebrachte Anliegen soweit zu bearbeiten, dass es als abgeschlossen betrachtet werden kann, oder der Coachee in der Lage ist, es ohne Hilfe des Coaches weiter zu verfolgen.

Der Bearbeitungsprozess eines Anliegens kann in Anlehnung an Grawe (1998) in einen Klärungs- und einen Umsetzungsteil zerlegt werden. Im Laufe der Klärung soll sich der Coachee über seine Bedürfnisse, sein relevantes (soziales) Umfeld, seine Rolle und seine Intentionen bewusst werden. Diese sollen in eine neue Perspektive gesetzt werden und aus dieser Perspektive soll der Coachee sich darüber klar werden, was er ändern kann und will (Grawe 1998). Abgeschlossen ist die Klärung, wenn der Coachee ein für ihn passendes Ziel formuliert hat. Dieses dient als verbindendes Scharnier zwischen den beiden Phasen. In der zweiten Phase erfolgt dann die Umsetzung des Zieles. Hier werden die einzelnen Schritte, die zur Zielerreichung oder Zielannäherung notwendig sind, im Detail geplant, erste Verhaltensschritte ausgeführt, Barrieren überwunden und erreichte Teilziele evaluiert. Wobei hier anzumerken ist, dass es durchaus Ziel eines Coachings sein kann, ein attraktives Zielbild (Kuhn 2014) zu erarbeiten und der Coachingprozess damit abgeschlossen ist. Ebenso kann der Entwurf einer Perspektive, oder der Zugewinn an Orientierung und Klarheit als Ziel eines Coachings betrachtet werden. Die 
zweite Phase endet, wenn der Coachee sein Ziel entweder erreicht hat, oder die Bearbeitung so weit fortgeschritten ist, dass er die Unterstützung des Coaches nicht mehr benötigt.

Mit fortschreitender Klärung des Anliegens ist zu erwarten, dass der Coachee größere Klarheit über seine Wünsche und Ziele (Zielklarheit) gewinnt, neue Sichtweisen auf sein Anliegen entwickelt, für ihn gangbare Lösungswege sichtbar werden (Perspektive), er mit dem Anliegen verbundene Unsicherheit abbaut und sich der Erwartungen seines sozialen Umfeldes (Rollenklarheit) klarer wird. Damit einher geht in der Regel die Verbesserung des mit dem Anliegen verbundenen Affekts (Befindlichkeit) und der Zuversicht resp. der Erfolgshoffnung (Schwarzer 2004).

\section{Inkongruenz als Ergebnisindikator für Coaching}

Das Konzept der Inkongruenz wurde von Grawe (1998) formuliert und ist in der Psychotherapie gut eingeführt. Für den Bereich Coaching wurde es in einer Prä-Post-Feldstudie eingesetzt (Künzli 2012). Von Inkongruenz spricht man, wenn Menschen ihre Ziele, z. B. aufgrund motivationaler Konflikte, fehlender Bewältigungsressourcen, ungünstiger Umweltbedingungen oder aufgrund des fehlenden Bewusstseins über die wirklichen Determinanten des eigenen Verhaltens und die eigenen Ressourcen (Grosse-Holtforth et al. 2004), nicht oder nur ungenügend realisieren können. Reichen die subjektiven oder objektiven Handlungsressourcen einer Person nicht mehr aus, um die aktivierten Ziele zu erreichen, wird sie bereichsspezifisch Unbehagen, Unklarheit, Unstimmigkeit, Angst, Gefühle von Autonomieverlust oder sogar Machtlosigkeit erleben. Dies kann der Moment sein, Unterstützung, z. B. ein Coaching, in Anspruch zu nehmen.

\section{Ziele und Kontext der Studie}

Das Ziel der Studie war der Nachweis der Wirksamkeit von Coachings, die nach der Vorgabe des Modells „Systemischkonstruktivistisches Einzel- und Teamcoaching im Management" SKETM, durchgeführt wurden. Erwartet wird, dass sich die Coachees ihren selbst gesetzten Zielen annähern, dass Fortschritte hinsichtlich der Klärung und der Umsetzung ihres Anliegens wahrnehmen und sich das Inkongruenzerleben verbessert und sich auf diesen Indikatoren signifikante Interaktions- und Zeiteffekte ergeben.

\section{Methode}

\subsection{Stichprobe}

Von den insgesamt 70 Coachees, die sich am Projekt beteiligten wurden sechs aufgrund inkonsistenter oder zu vieler fehlender Daten (z. B. Ausfüllen der Fragebögen von t0 und t1 zur selben Zeit; mehr als $80 \%$ fehlende Daten) von der Analyse ausgeschlossen. Das durchschnittliche Alter der verbleibenden 64 Coachees lag bei 41,58 (SD = 10,37) Jahren, wobei der jüngste Coachee 23 und der älteste 63 Jahre alt waren. Die Stichprobe setzte sich aus 24 Frauen $(37,50 \%)$ und 33 Männern $(51,56 \%)$ zusammen (7 ohne Angaben). Einunddreißig (47,4\%) waren in einer Führungsfunktion in der Linie, 5 (7,8\%) in einer Projektleitungspositionen, $3(4,7 \%)$ arbeiteten in einer Stabstelle, $10(15,6 \%)$ waren zum Zeitpunkt des Coachings selbständig, $13(20,3 \%)$ hatten keine Führungsposition und 7 (10,9\%) machten keine Angaben zu Führungsfunktionen. Die Latenzzeit, das heißt die Zeitdauer während derer sich die Coachees mit ihrem Anliegen beschäftigten, bevor sie ein Coaching in Anspruch nahmen betrug durchschnittlich $16,06(S D=20,99)$ Monate.

Die Coachees wurden durch insgesamt 25 Coaches, die nach dem Modell der Ausbildung ,Systemisch-konstruktivistisches Einzel- und Teamcoaching im Management" „SKETM (Meier 2014) arbeiten, angeworben. Von den Coaches waren 7 weiblich und 15 männlich (3 ohne Angaben). Das Durchschnittsalter der Coaches betrug 47,9 $(S D=10,08$; Range 32-69) Jahre und sie hatten durchschnittlich 6,9 (SD = 6,75; Range 0,5-25) Jahre Erfahrung als Coach.

\subsection{Design}

In der Studie wurde ein quasiexperimentelles Wartegruppendesign mit drei Messzeitpunkten verwendet (Tab. 1). Auf eine randomisierte Zuteilung musste, auch als Konzession an die Praxis, verzichtet werden. Eine a-priori durchgeführte Poweranalyse zeigte, dass auf der Erstmessung ca. 70 Coachees benötigt würden, um mittlere Effekte zu entdecken. Um die möglichen Einflussvariablen Alter, Erfahrung und Geschlecht des Coachs konstant zu halten, war ursprünglich vorgesehen, über einen Aufruf an alle ehemaligen Absolventen der Ausbildung SKETM insgesamt 35 Coaches zu gewinnen, die je zwei Coachees, je einen für die Interventions- und einen für die Wartekontrollgruppe, anwerben würden. Dies konnte nicht realisiert werden.

Tab. 1 Studiendesign

\begin{tabular}{|c|c|c|c|c|c|}
\hline Messzeitpunkt & t0 & & $\mathrm{t} 1$ & & t2 \\
\hline$\overline{\mathrm{IG}}$ & $\mathrm{O}$ & 1 Tag Coaching & $\mathrm{O}$ & ca. 4 Wochen & $\mathrm{O}$ \\
\hline WG & $\mathrm{O}$ & ca. 4 Wochen & $\mathrm{O}$ & 1 Tag Coaching & $\mathrm{O}$ \\
\hline
\end{tabular}


Schlussendlich nahmen 25 Coaches teil, die zwischen 1 bis 9 Coachees für die Projektteilnahme gewinnen konnten. Die Coaches informierten neu anfragende Coachees über das Projekt und teilten sie bei Einwilligung zur Projektteilnahme der Interventions- (IG) oder der Wartekontrollgruppe (WG) zu. Die Zuteilungsentscheidung wurde durch die Coaches getroffen. Die Coachees der IG erhielten ihr Coaching sofort nach Anmeldung. Die Aufnahme der Coachees der WG wurde um ca. vier Wochen aufgeschoben. Die Coachees füllten insgesamt drei Fragebögen aus. Den ersten kurz nach der Anmeldung, den zweiten entweder nach (IG) oder unmittelbar vor dem Coaching (WG). Die dritte Erhebung fand vier Wochen nach (IG) oder unmittelbar nach dem Coaching statt. Aufgrund fehlender Werte schwankt die Anzahl der gültigen Werte auf Skalenebene über die Messzeitpunkte und die Gruppen $\left(n_{t 0}=53-57 ; n_{t l}=50-53\right.$; $n_{t 2}=45-46$ ). Eine Ausnahme dazu bildet die Skala Rollenklarheit mit wesentlich tieferen gültigen Werten. Grund dafür war, dass die Items neben der sechsstufigen Skala auch noch die Antwort In Hinsicht auf mein Anliegen nicht relevant zuließen. Diese Antwortoption wurde häufig gewählt, was dazu führte, dass auf Skalenebene nur eine geringe Anzahl auswertbarer Datensätze zur Verfügung standen $\left(n_{t 0}=\right.$ $25 ; n_{t 1}=31 ; n_{t 2}=25$ ).

Da die transparente und strikte Umsetzung des Prozesses im SKETM als bedeutendster Wirkfaktor betrachtet wird, wurde für die Behandlungstreue ein aus 8 Items bestehender ad-hoc-Index entworfen. Die 8 Items repräsentieren aus Sicht des Entwicklers des SKETM die wichtigsten Komponenten des Prozesses (Beispiel Item: „Mein Coach hat den mir bekannten Ablauf des Coachings in vollem Umfang eingehalten"; sechsstufige Skala von $1=$ trifft nicht $z u$ bis $6=$ trifft vollständig $z u$ ). Der Behandlungstreue-Index wurde als Kovariate ins Modell aufgenommen $(\alpha=0,82)$.

Die Datenerhebung wurde mit dem Survey-Tool Unipark durchgeführt.

\subsection{Intervention}

Die Coachings wurden nach der Konzeption der Ausbildung ,Systemisch-konstruktivistisches Einzel- und Teamcoaching im Management" SKETM (Meier 2013, 2014) durchgeführt. Das genaue Vorgehen ist in Meier (2013, 2014) beschrieben. Für die Versuchsdurchführung wurden keine Anpassungen am Interventionsmanual (Meier 2013, 2014) durchgeführt. Ein Coaching nach den Vorgaben der SKETM dauert in der Regel zw. 6-8 h und wird innerhalb eines Tages durchgeführt. Das Angebot des SKETM gegenüber dem Coachee besteht in der Lösung des anstehenden Veränderungsthemas und dem Erlernen des Prozesses durch den Coachee, damit er, im Sinne der nachhaltigen Selbstorganisation, sich in Zukunft ohne fremde Unterstützung verändern (coachen) kann. Als entscheidender Wirkfaktor wird im SKETM die transparente und strikte Anwendung des vorgegebenen Prozesses betrachtet. Dies bedeutet, dass der Coach dem Coachee zu Beginn des Prozess die einzelnen Phasen aufzeigt und erläutert. Der Abschluss einer Phase, Verzweigungen innerhalb der Phasen und Teilschritte werden während des Prozesses erklärt. Der Coach ist der sogenannte „Diener“ des Prozesses, da er selbst oder aus sich selbst heraus keine situativen Interventionen entwickelt und initiiert. Er moderiert den Coachingprozess und achtet dabei darauf, dass die definierten fixen und variablen Bestandteile im Coachingverlauf ihre richtige Anwendung erfahren. Der Ablauf des Prozesse, seine fixen und variablen Interventionen werden in der Ausbildung zum SKETMCoach vermittelt und sind verschriftlicht (Meier 2014). Basis für die Methode sind zwei Haltungen, die aus dem Konstruktivismus und dem systemische Denken abgeleitet sind:

Konstruktivismus - Realität ist immer eine Konstruktion der Person, die Realität beschreibt. Eine objektive Abbildung einer wie auch immer gearteten Realität existiert nicht. Daraus folgt, dass der Coachee allein das Recht hat, seine Realität zu deuten.

Systemisches Denken - Menschliche Erlebens- und Verhaltensweisen vollziehen sich immer in Zusammenhang mit anderen Menschen und Umweltbedingungen. Systeme stehen immer in Wechselwirkung zueinander und beeinflussen sich gegenseitig. Systeme können nicht objektiv beschrieben werde (vgl. Konstruktismus). Die Beschreibung und Interpretation von relevanten Systemen kann daher nur vom Coachee vorgenommen werden.

Aus diesen Haltungen werden alle Prozesselemente abgeleitet (Meier 2013, 2014). Dieser beruht auf drei Grundannahmen und ist in fünf Phasen gegliedert.

Der Coachingprozess ...

- ist eine fest definierte Methode, die vom Coach einzuhalten ist.

- ist vom Coach durch die Werte Freiheit (der Coachee kann zwischen von ihm erkannten Alternativen entscheiden), Freiwilligkeit (der Coachee kann seine Veränderungsthematik und den Zeitpunkt der Veränderung entscheiden), Ressourcenverfügung (der Coachee verfügt über und hat Zugriff auf Ressourcen, die zur Selbstorganisation und Veränderungsrealisierung benötigt werden) und Selbststeuerung (der Coachee kann Veränderungsanforderungen selbst erkennen und realisieren) im konsequenten Blick auf den Coachee wahrzunehmen.

- löst beim Coachee Wahrnehmungserweiterung(-veränderungen) aus, ermöglicht ihm die Entwicklung von Handlungsalternativen und verbessert seine situative und zukünftige Entscheidungsfähigkeit.

Phase 1 - Kontrakt und Kontakt Der Coach erläutert dem Coachee sein Coachingverständnis und den damit verbundenen Verantwortlichkeiten im Prozess und den gewoll- 


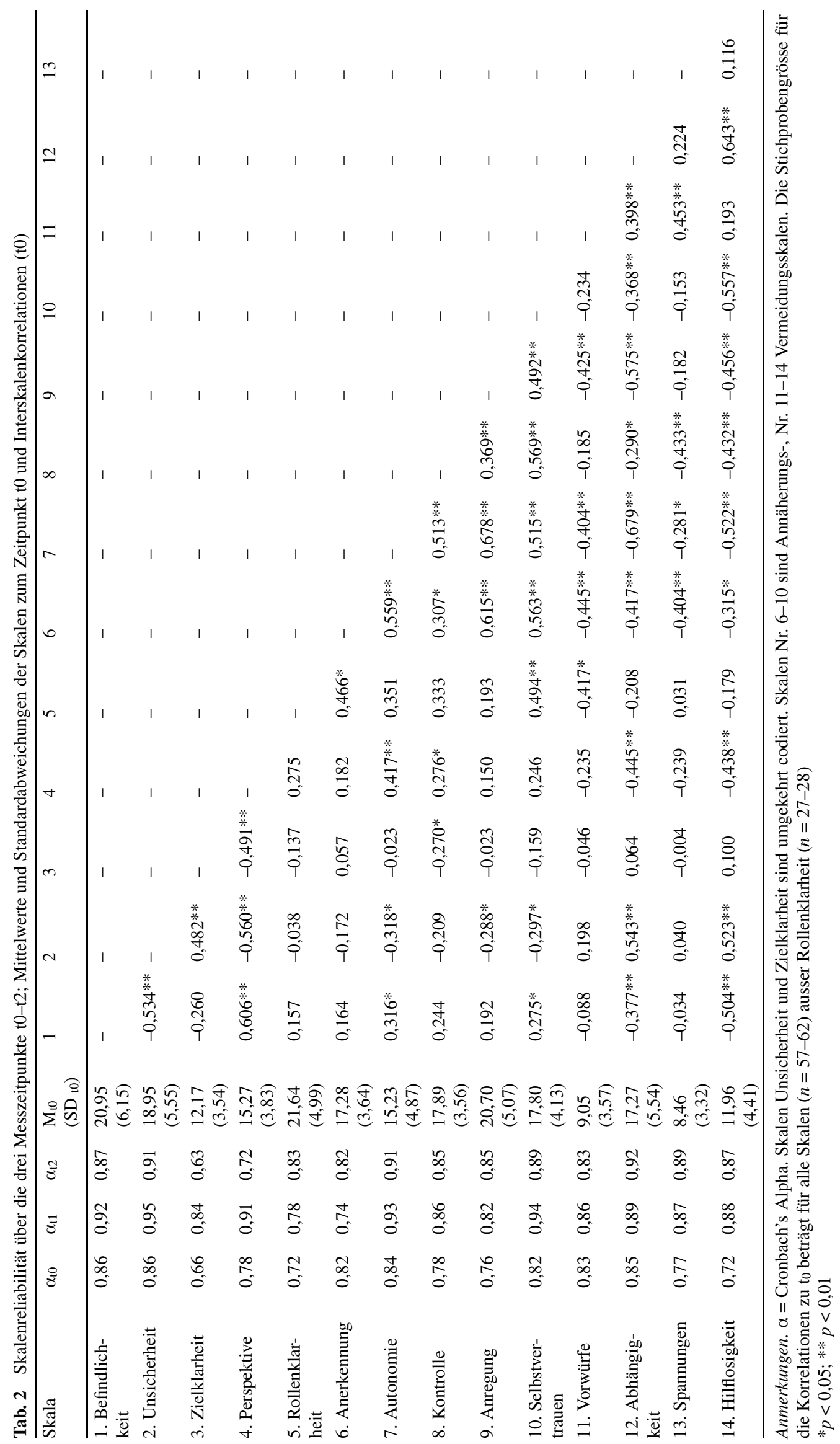


ten Folgen (nachhaltige Selbstorganisation). Absicht ist eine bewusste Vertragsgestaltung zwischen Coach und Coachee.

Phase 2 - Themen- und Zielklärung Das Veränderungsthema wird aus Sicht und durch den Coachee visualisiert, mit der Absicht, die konkrete und emotional gewichtete Bedeutung seines Veränderungsthemas in einem spezifischen Kontext deutlich werden zu lassen.

Phase 3 - Zielorientierte Ressourcenidentifikation und Reflexion Auf der Basis des Kompetenzmodells werden in den Bereichen fachlich-methodische Kompetenz, soziokommunikative Kompetenz, persönliche Kompetenz und Feldkompetenz konkrete Ressourcen zur Zielerreichung identifiziert. Ein wichtiges Merkmal bei der Ressourcenidentifikation ist die Unterscheidung in hilfreiche und hinderliche Ressourcen, über die der Coachee verfügt.

Phase 4 - Entwicklung eines Handlungsplans zur Zielerreichung Eventuelle Handlungsbarrieren und Umsetzungsschwierigkeiten werden identifiziert und thematisiert und der Coachee entscheidet, welche Ressourcen und Kompetenzen er einsetzen will. Des Weiteren werden Kriterien definiert, anhand derer der Coachee den Handlungsfortschritt überprüfen kann.

Phase 5 - Definition von Controlling Maßnahmen und Sicherung der Nachhaltigkeit $\mathrm{Im}$ Ergebnis sollen die Controlling Maßnahmen einerseits dazu dienen, zu erkennen, ob die geplanten Handlungen qualitativ und quantitativ zum vereinbarten Erfolg geführt haben. Auf der anderen Seite soll in dieser Phase aber auch die nachhaltige Handlungskompetenz gesichert werden. Es gilt herauszufinden und festzulegen, welche vergleichbaren Themen in anderen Kontexten in Zukunft durch den Coachee selbstorganisiert realisiert werden können.

\subsection{Messinstrumente}

In Tab. 2 sind die Skalenreliabilitäten aller Skalen über die drei Messzeitpunkte sowie die Interskalenkorrelation für den ersten Messzeitpunkt aufgeführt.

Zielannäherung Zur Messung der Zielannäherung wurde ein vereinfachtes, 11-stufiges Goal Attainment Scaling (Kiresuk und Sherman 1968) verwendet $(0=$ maximale Distanz zur Zielerreichung; 10 = vollständige Zielerreichung).

Fragebogen Evaluation Coaching - Klärung und Umsetzung (FEC-KU) Zur Erfassung der subjektiv wahrgenommen Klärung und Umsetzung des Anliegens wurde der Fragebogen Evaluation Coaching - Klärung und Umsetzung FEC-KU (Künzli, in Vorbereitung) eingesetzt. Der
FEC-KU umfasst in der verwendeten Form 24 Items und misst den Fortschritt der Klärung und Umsetzung eines Anliegens auf fünf Skalen: Befindlichkeit (6 Items), Zielklarheit (4 Items), Perspektive (4 Items), Sicherheit (5 Items) und Rollenklarheit (5 Items). Die Skalen des FEC-KU weisen tiefe bis gute interne Konsistenzen über die drei Messzeitpunkte aus. Die einzelnen Items nehmen sprachlich Bezug auf das Anliegen, sind aber vom konkreten Inhalt des Anliegens unabhängig. Ein Beispielitem aus der Skala Perspektive lautet: „Wenn ich an mein Anliegen denke, sehe ich bereits erste Lösungsmöglichkeiten“ (6-stufige Skala von $1=$ trifft überhaupt nicht $z u$ bis $6=$ trifft vollständig $z u)$.

Die Items der Skalen wurden im Wesentlichen aus dem Feld abgeleitet. In Workshops mit Coachs und Laufbahnberatern und in Gesprächen mit Coachees wurde herausgearbeitet, wie sich Coachees nach einem Coaching oder einer Laufbahnberatung (Künzli und Zihlmann 2009) in Hinsicht auf ihr Anliegen Äußern. Diese Aussagen wurden zu Skalen zusammengefasst und für den Bereich der Laufbahnberatung einer faktoriellen Überprüfung unterzogen (Künzli und Zihlmann 2009). Die für die Laufbahnberatung erarbeiteten Skalen wurden dann in adaptierter Form auch im Coaching verwendet. Eine Überprüfung der Skalen des FEC-KU steht noch aus.

Die Skala Befindlichkeit wurde in Anlehnung an die PANAS-Skala (Watson et al. 1988) erstellt. Im Unterschied zur PANAS-Skala gibt die Skala Befindlichkeit Auskunft über die unmittelbar mit dem Anliegen verbundene und nicht über die generelle Affektlage. Löst das Nachdenken über sein Anliegen beim Coachee eher unangenehme, bedrohliche oder selbstabwertende Gefühle aus, kann dies als Hinweis für tiefe oder fehlende auf das Anliegen bezogene Wirksamkeitserwartungen und Bewältigungskompetenzen gedeutet werden. Empfindet der Coachee in Bezug auf sein Anliegen hingegen angenehme Gefühle oder freut sich sogar auf die damit verbundenen Herausforderungen, lässt dies darauf schließen, dass er über hinreichende Bewältigungskompetenzen verfügt (Schwarzer 2001). Die Reliabilitäten über die drei Messzeitpunkte können als gut bezeichnet werden. Beispiel-Item: „Wenn ich an mein Anliegen denke, fühle ich mich entspannt.“

In der Skala Zielklarheit kommt zum Ausdruck, ob der Coachee in Hinsicht auf sein Anliegen schon über ein (klares) Ziel verfügt. Über ein Ziel zu verfügen, und dies auch zu wissen, ist ein zentrales Element der subjektiven Handlungskontrolle (Flammer 1990). Zu Beginn eines Coachings ist Zielunklarheit eher die Regel als die Ausnahme. Meistens können Coachees einen Zielbereich aber noch kein konkretes Ziel formulieren. Die Reliabilität der Skala bewegt sich im tiefen ( $\mathrm{t} 0$ und $\mathrm{t} 2$ ) bis akzeptablen Bereich ( $\mathrm{t} 1$ ). Beispiel-Item: „Wenn ich an mein Anliegen denke, habe ich konkrete Vorstellungen davon, was ich will.“ 
Ein weiteres wichtiges Element der Handlungskontrolle ist das Wissen über Wege, die zielführend sind (Flammer 1990). Dies kommt in der Skala Perspektive zum Ausdruck. Die Reliabilitäten für die Skala Perspektive bewegen sich im akzeptablen Bereich. Beispiel-Item: ,Wenn ich an mein Anliegen denke, bin ich überzeugt, dass ich auf dem richtigen Weg bin."

Die Skala Unsicherheit ist Ausdruck für steigende $\mathrm{Zu}$ versicht und Hoffnung bei der fortschreitenden Klärung des Anliegens. Tiefe Werte sind Ausdruck dafür, dass Aspekte des Anliegens, seien es Variablen des Umfeldes, mögliche Lösungswege und/oder Ziele, noch nicht hinreichend geklärt sind. Die wahrgenommenen Unstimmigkeiten lassen Zweifel entstehen und man zögert, weitere Schritte in die Hand zu nehmen. Hohe Werte weisen darauf hin, dass der Weg nun frei ist, nächste Schritte eingeleitet werden können, der Lösungsweg und/oder das Ziel zum Coachee passen. Die Reliabilitäten bewegen sich in einem akzeptablen bis guten Bereich. Beispiel-Item: ,Wenn ich an mein Anliegen denke, zweifle ich daran, ob die Lösungsmöglichkeiten, die mir durch den Kopf gehen, wirklich in Frage kommen“.

Die Skala Rollenklarheit trägt dem Umstand Rechnung, dass der Coachee, und damit auch die von ihm angestrebten Ziele und seine Lösungswege, in Passung mit den Erwartungen aus seinem sozialen Umfeld stehen müssen. Die Rolle wird dabei als Bündel von Erwartungen verstanden, das vom Umfeld an den Coachee herangetragen wird. Die Reliabilitäten bewegen sich über die drei Messzeitpunkte im akzeptablen Bereich. Beispiel-Item: „Wenn ich an mein Anliegen denke, ist mir klar, was mein Umfeld von mir erwartet."

Inkongruenz Zur Messung der Inkongruenz wurden Teile des Inkongruenzfragebogens INK (Grosse-Holthfort et al. 2004) eingesetzt. Der INK enthält im Original 94 Items und erfasst den Grad unzureichender Umsetzung motivationaler Ziele (Zufriedenheit mit der Umsetzung von Annäherungszielen bzw. Nichteintreffen von Vermeidungszielen). Um den Fragebogen so kurz wie möglich zu gestalten, wurden für den vorliegenden Zweck die ursprünglich 14 Annäherungs- resp. 9 Vermeidungsskalen auf 5 (Anerkennung, Kontrolle, Selbstvertrauen, Autonomie, Anregung (im Original „Das Leben auskosten“)), resp. 4 (Vorwürfe Kritik, Spannungen mit anderen, Abhängigkeit/Autonomieverlust, Hilflosigkeit/Ohnmacht) verkürzt. Entfernt wurden Skalen, die entweder nicht auf den Kontext von Business-Coaching passten (z. B. Intimität/ Bindung; Glauben/Sinn; Sich verletzbar machen; Alleinsein/Trennung), oder aufgrund inhaltlicher Überlegungen redundant erschienen (z. B. Versagen vs. Vorwürfe/Kritik). Auf die Berechnung und den Vergleich der Gesamtskalen „Inkongruenz Annäherungsziele“, „Inkongruenz Vermeidungsziele“ und „Gesamtinkongruenz“ musste daher ver- zichtet werden. Um die bei einer ungeraden Anzahl von Antwortmöglichkeiten für Probanden oft schwer zu interpretierende mittlere Position zu vermeiden (Mummendey und Grau 2008), und um das Antwortformat einheitlich zu halten, wurden in Abweichung zum Original 6- und nicht 5-stufige Likertskalen verwendet (Annäherungsskalen: 1 = viel zu wenig; 6 = völlig ausreichend; Vermeidungsskalen: $1=$ trifft überhaupt nicht $z u ; 6=$ trifft sehr stark $z u) . \mathrm{Ob}$ sich diese Streckung auf die Kennwerte der Skala auswirkt, konnte aus den vorliegenden Daten nicht ermittelt werden. Dass dies nicht der Fall sein muss, zeigte Leung (2011) anhand der Rosenberg Self-Esteem Scale. Er konnte belegen, dass die Verwendung einer 4-, 5-, 6 oder 11-stufigen Skala keinen Einfluss auf die Mittelwerte, die Standardabweichungen, die Item-Item Korrelationen, die Item-Total Korrelationen, das Cronbach's Alpha und die Faktorladungen hatte. Die sechs- und die 11-stufigen Skalen näherten sich hingegen eher der Normalverteilung an und zeigten tendenziell eine geringere Schiefe und Kurtosis. Die Reliabilitäten aller Skalen über die drei Messzeitpunkte sind zufriedenstellend.

\section{Statistische Analyse}

Da es sich um einen Zweigruppenplan mit Messwiederholungen handelte, wurden alle Ergebnisvariablen mit der Prozedur Generalized Estimating Equations GEE (Zeeger et al. 1988) überprüft. Der Vorteil der Prozedur GEE im Vergleich zur Varianzanalyse mit Messwiederholung liegt zum einen darin, dass die bei Längsschnittdaten auftretenden Korrelationen zwischen den Messzeiten in der Analyse mit berücksichtigt werden. Zum anderen werden in GEE auch unvollständige Datensätze in der Analyse berücksichtigt. Die fehlenden Daten werden über eine Schätzgleichung ersetzt.

Die beste Passung mit den Daten der Ergebnisindikatoren (INK, Zielannäherung, FEC-KU) wurde mit der Gamma-Verteilung mit Log Link-Funktion erreicht. Um Einschränkungen der Kovarianzstruktur zu vermeiden, wurde von unstrukturierten Korrelationen zwischen den Messzeitpunkten ausgegangen. Um Ausreißereffekte und die Auswirkung von einflussreichen Beobachtungen $\mathrm{zu}$ reduzieren wurde ein robuster Schätzer (Huber sandwich estimator; Huber 1967; White 1980) verwendet. Dies entspricht dem Standardvorgehen bei Anwendung der Prozedur GEE. Achsabschnitt und Steigungskoeffizienten wurden in allen Analysen berücksichtigt. Dies gilt als Standardvorgehen bei der Modellierung von Längsschnittdaten (Twisk 2013). Die Messwerte des Messzeitpunktes t0 werden dabei als Eingangswerte verwendet und die Steigung entspricht dem linearen Zuwachs der Messwiederholung. Zur Korrektur der Korrelation zwischen den Messzeitpunkten innerhalb 
Tab. 3 Deskriptive Angaben Interventionsgruppe IG und Wartekontrollgruppe WG Zeitpunkt t0

\begin{tabular}{|c|c|c|c|}
\hline Variablen & IG t0 $\left(\mathrm{SE}^{\mathrm{a}}\right)$ & WG t0 $\left(\mathrm{SE}^{\mathrm{a}}\right)$ & \\
\hline$\overline{\text { Geschlecht Coach (w) }}$ & $11(21 \%)$ & $12(23 \%)$ & Fishers exakter Test; $p=0,525$ \\
\hline Alter Coach & $42,52(1,57)$ & $45,50(2,07)$ & $\mathrm{T}(46) ; p=0,259$ \\
\hline Erfahrung Coach (Jahre) & $6,09(1,06)$ & $8,64(1,44)$ & $\mathrm{T}(53) ; p=0,167$ \\
\hline Geschlecht (w) & $17(27 \%)$ & $7(11 \%)$ & Fishers exakter Test; $p=0,003^{* *}$ \\
\hline Alter Coachee & $41,67(2,0)$ & $41,71(1,82)$ & $\mathrm{T}(53) ; p=0,987$ \\
\hline Latenzzeit Coachee & $16,61(3,55)$ & $15,55(3,90)$ & $\mathrm{T}(62) ; p=0,841$ \\
\hline Zielannäherung & $3,69(0,34)$ & $3,89(0,30)$ & Wald; $p=0,661$ \\
\hline FEC-KU Befindlichkeit & $20,74(1,10)$ & $20,84(1,15)$ & Wald; $p=0,951$ \\
\hline FEC-KU Sicherheit & $19,21(1,05)$ & $19,25(1,08)$ & Wald; $p=0,978$ \\
\hline FEC-KU Zielklarheit & $11,84(0,67)$ & $12,51(0,67)$ & Wald; $p=0,478$ \\
\hline FEC-KU Rollenklarheit & $22,19(1,53)$ & $21,42(1,30)$ & Wald; $p=0,710$ \\
\hline FEC-KU Perspektive & $15,64(0,77)$ & $14,93(0,67)$ & Wald; $p=0,488$ \\
\hline INK Anerkennung & $4,06(0,20)$ & $4,55(0,11)$ & Wald; $p=0,036^{*}$ \\
\hline INK Autonomie & $3,94(0,22)$ & $3,60(0,22)$ & Wald; $p=0,260$ \\
\hline INK Kontrolle & $4,53(0,15)$ & $4,28(0,18)$ & Wald; $p=0,290$ \\
\hline INK Anregung & $4,08(0,19)$ & $4,24(0,17)$ & Wald; $p=0,519$ \\
\hline INK Selbstvertrauen & $4,54(0,19)$ & $4,18(0,18)$ & Wald; $p=0,177$ \\
\hline INK Vorwürfe & $3,20(0,25)$ & $2,94(0,19)$ & Wald; $p=0,398$ \\
\hline INK Abhängigkeit & $3,69(0,22)$ & $3,55(0,22)$ & Wald; $p=0,659$ \\
\hline INK Spannungen & $2,30(0,17)$ & $2,02(0,17)$ & Wald; $p=0,183$ \\
\hline INK Hilflosigkeit & $3,21(0,20)$ & $2,87(0,20)$ & Wald; $p=0,224$ \\
\hline
\end{tabular}

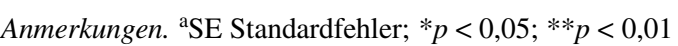

der Versuchspersonen und um die Veränderungen über die Messzeitpunkte in Relation zur Gruppenzuteilung zu überprüfen, wurde der Steigungskoeffizient auch als Interaktionseffekt mit der Gruppenvariable modelliert (Intervention * Messzeitpunkt). Zur Bewertung der Effekte zwischen den Gruppen wurden die Mittelwerte der IG und der WG zum Zeitpunkt t1 verglichen. Die Effekte innerhalb der Gesamtgruppe wurden über Mittelwerte der Zeitpunkte t0 und t2 berechnet. Zur Bewertung der Veränderungen wurde die Effektgrösse $d$ (Cohen 1988) verwendet. Die Veränderungen wurden mit der Prä-Streuung der Gesamtgruppe verglichen. Alle Analysen wurden mit SPSS 23 durchgeführt.

\section{Qualitative Auswertungen}

Zur Ergänzung der quantitativen Veränderungsmessung wurde den Coachees zum Zeitpunkt t1 (IG), resp. t2 (WG) eine offene Frage nach Veränderungen gestellt, die sie subjektiv auf das Coaching zurückführten. Die Antworten wurden nach Mayring (2010) ausgewertet. Die Kategorienbildung geschah induktiv.

\section{Ergebnisse}

In Tab. 3 sind die Mittelwerte und Standardfehler der PräWerte (t0) aller Variablen der Interventions- und der Warte- kontrollgruppe aufgeführt. Frauen sind in der Interventionsgruppe signifikant häufiger vertreten als in der WG und die Skala Anerkennung der WG weist auf der Baseline einen signifikant höheren Mittelwert aus als die IG. Generell fällt auf, dass die WG auf den INK-Skalen leicht höhere Baseline-Werte ausweist.

In Tab. 4 sind die geschätzten Werte für die drei Messzeitpunkte und die beiden Gruppen, sowie die Überprüfungen des Interaktionseffekts von t0 auf $\mathrm{t} 1$ und des Zeiteffektes von t0 auf t 3 aufgeführt. Tab. 5 zeigt Einzelvergleiche der Veränderungen von $\mathrm{t} 1$ auf $\mathrm{t} 2$ der IG (Nachhaltigkeit). Es wurde davon ausgegangen, dass sich die Werte der Ergebnisvariablen der Interventionsgruppe zum Zeitpunkt t1 im Vergleich zur Wartekontrollgruppe durch das Coaching erhöhen (FEC-KU: Zielerreichung, Befindlichkeit, Perspektive, Rollenklarheit; INK: Selbstvertrauen, Kontrolle, Anregung, etc.) oder vermindern (FEC-KU: Zielklarheit, Unsicherheit; INK: Vorwürfe, Abhängigkeit, Spannungen, Hilflosigkeit). Auf dem Messzeitpunkt 12 sollten die Werte der beiden Gruppen gleichauf, aber höher liegen als zum Messzeitpunkt t0. Der Behandlungstreue-Index zeigte einen starken Deckeneffekt bei geringer Varianz, hoher Kurtosis und Schiefe $(M=5,62, S D=0,50$, Kurtosis $=3,106$, Schiefe $=$ $-1,844)$ und wurde daher nicht in das Modell aufgenommen.

Zielannäherung Die Zielannäherung nimmt bei beiden Gruppen über die Zeitpunkte t0-t2 signifikant $\mathrm{zu}(d=$ 


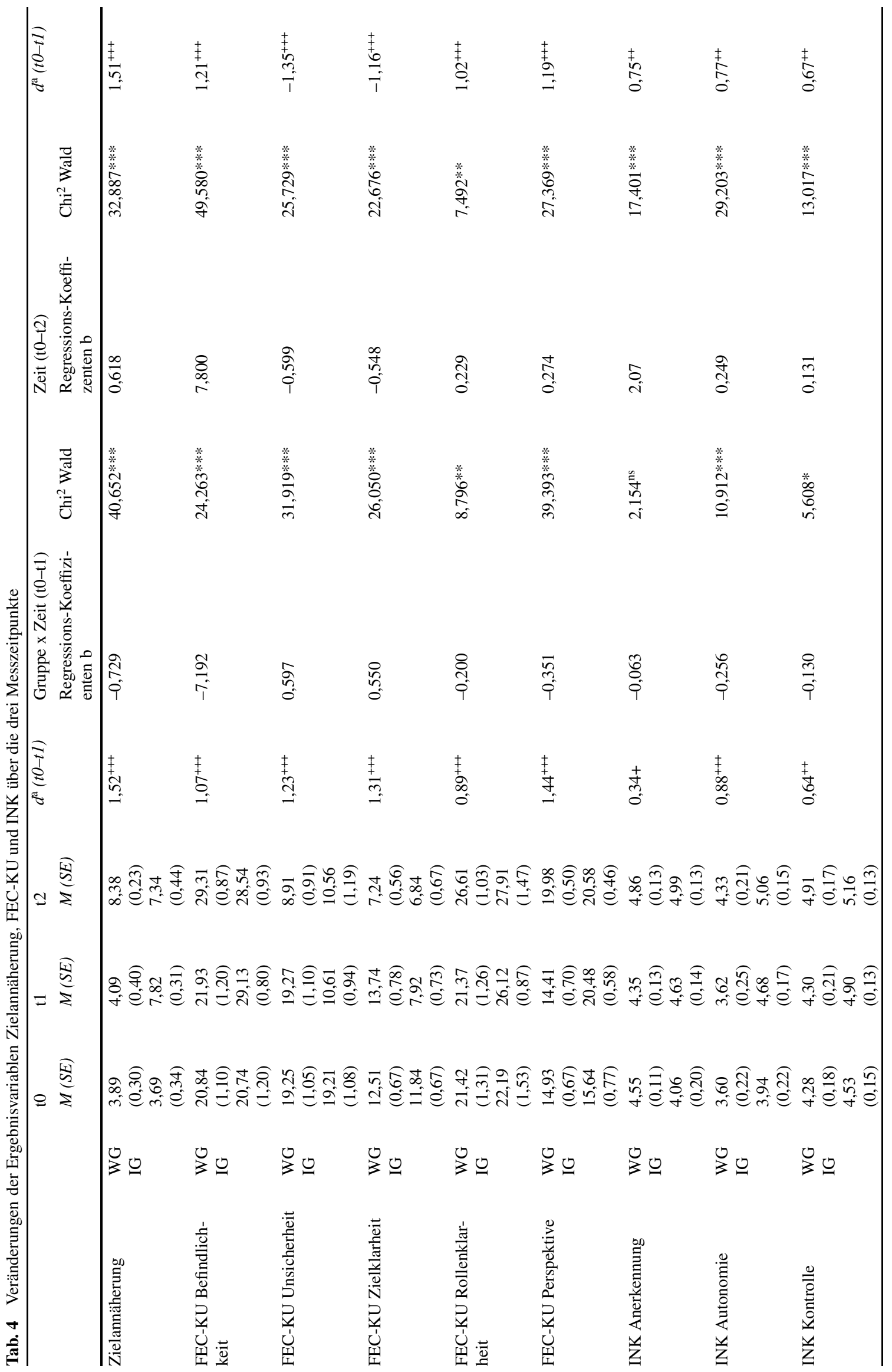




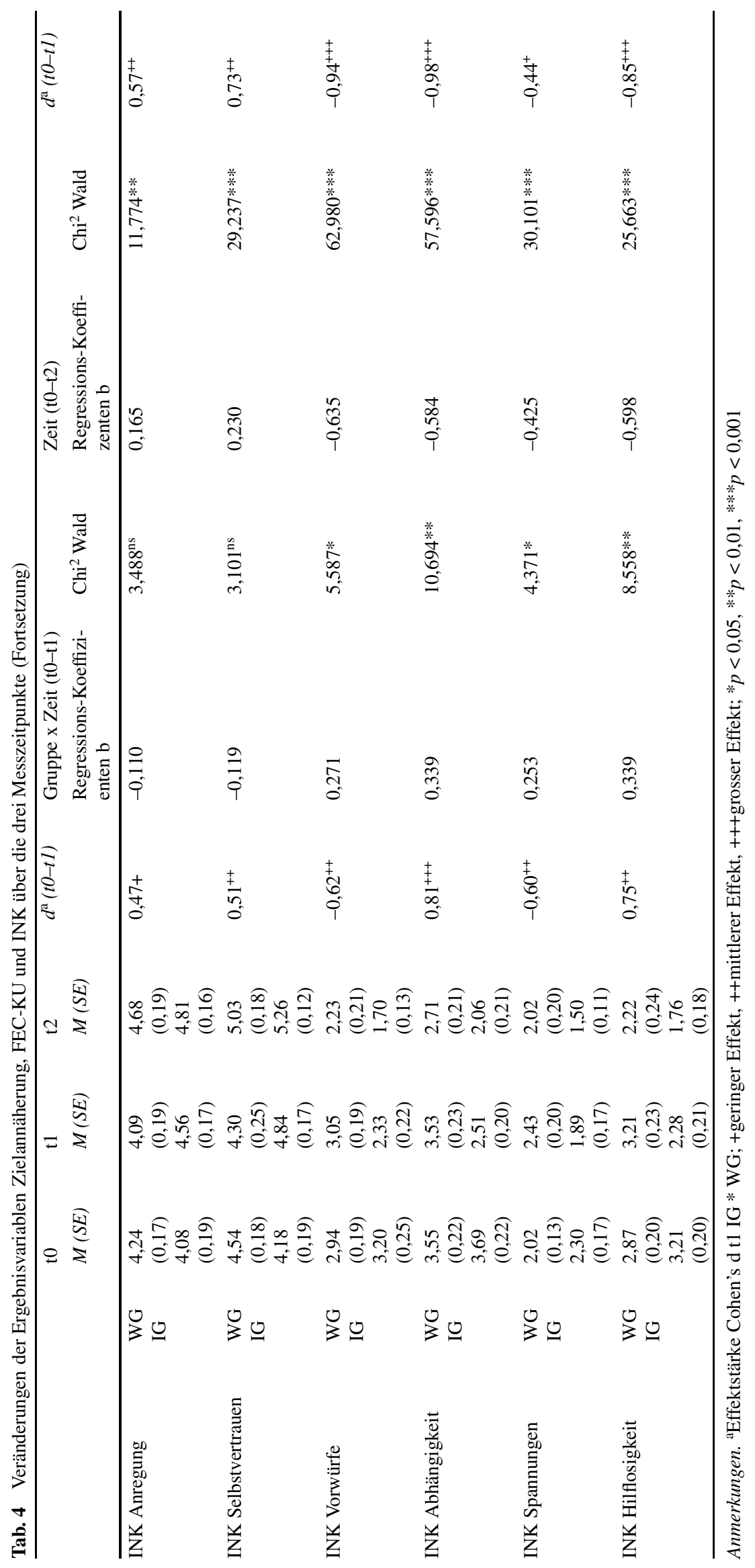


Tab. 5 Einzelvergleiche Veränderungen Interventionsgruppe IG von t1 auf t2 (Nachhaltigkeit)

\begin{tabular}{llllll}
\hline & Mittlere & SE $^{\mathrm{a}}$ & $p^{*}$ & \multicolumn{2}{c}{$95 \%$ Wald-Konfidenzintervall } \\
& Diff & & & 0,225 & $-0,355$ \\
Zielannäherung & 0,578 & 0,476 & 0,482 & $-0,845$ & 1,510 \\
FEC-KU Befindlichkeit & 0,473 & 0,673 & 0,775 & $-2,094$ & 1,792 \\
FEC-KU Sicherheit & $-0,267$ & 0,932 & 0,166 & $-0,414$ & 1,561 \\
FEC-KU Zielklarheit & 0,996 & 0,719 & 0,114 & $-4,150$ & 2,405 \\
FEC-KU Rollenklarheit & $-1,854$ & 1,171 & 0,812 & $-0,884$ & 0,442 \\
FEC-KU Perspektive & $-0,096$ & 0,402 & 0,011 & $-0,779$ & 0,693 \\
INK Anerkennung & $-0,438$ & 0,173 & 0,012 & $-0,669$ & $-0,099$ \\
INK Autonomie & $-0,376$ & 0,149 & 0,018 & $-0,485$ & $-0,084$ \\
INK Kontrolle & $-0,265$ & 0,112 & 0,227 & $-0,636$ & $-0,045$ \\
INK Anregung & $-0,242$ & 0,201 & 0,015 & $-0,769$ & 0,151 \\
INK Selbstvertrauen & $-0,425$ & 0,175 & 0,003 & 0,220 & $-0,082$ \\
INK Vorwürfe & 0,630 & 0,209 & 0,020 & 0,071 & 1,040 \\
INK Abhängigkeit & 0,455 & 0,196 & 0,000 & 0,171 & 0,839 \\
INK Spannungen & 0,386 & 0,110 & 0,023 & 0,073 & 0,603 \\
INK Hilflosigkeit & 0,521 & 0,228 & & 0,969 \\
\hline
\end{tabular}

Anmerkungen. ${ }^{\text {aStandardfehler }}$

Signifikanz $* p<0,05, * * p<0,01, * * * p<0,001$

1,51). Die Auswertung über die Messzeitpunkte t0 und t1 zeigt einen signifikanten Interaktionseffekt für die Faktoren Messzeitpunkt und Gruppe. Die IG weist auf $\mathrm{t} 1$ eine höhere Zielerreichung aus als die WG. Die Effektstärke beträgt $d=$ 1,52. Die Resultate der IG bleiben vom Messzeitpunkt t2 auf t3 praktisch stabil. Die WG erreicht nach Abschluss ihres Coachings die gleichen Werte wie die IG.

\section{Fragebogen Evaluation Coaching - Klärung und Umset-} zung Die Werte aller Skalen des FEC-KU zeigen einen signifikanten Zeiteffekt von t0 auf t2. Die Effekte bewegen sind alle hoch $(d=1,02-1,35)$. Die Auswertungen über die Messzeitpunkte t0 und t1 über alle Skalen des FEC-KU zeigen zudem signifikante Interaktionseffekte für die Faktoren Zeitpunkt und Gruppe (t0-t1). Die Effektstärken $(d=$ 0,89-1,44) sind hoch. Auch hier bleiben die Resultate der IG vom Messzeitpunkt t2 auf t3 entweder stabil oder steigen leicht an. Die WG erreicht nach dem Abschluss ihres Coachings ähnliche Werte wie die IG.

Inkongruenz Die Werte aller Inkongruenzskalen zeigen einen signifikanten Zeiteffekt von t0-t2. Die Werte aller Annäherungsskalen sind zum Messzeitpunkt t2 höher als zum Messzeitpunkt t0 und die Werte der Vermeidungsskalen nehmen vom Messzeitpunkt t0 auf t2 ab. Mit Ausnahme der Skala Spannungen $(d=0,44)$ bewegen sich die Effekte im mittleren bis hohen Bereich $(d=0,57-0,94)$. Abgesehen von den Skalen Anerkennung, Anregung und Selbstvertrauen zeigen die Auswertungen über die Messzeitpunkte t0 und t1 signifikante Interaktionseffekte für die Faktoren Gruppe und Zeitpunkt für alle Skalen. Der Effekt für die
Skala Anregung $(d=0,47)$ ist klein bis mittel, diejenigen der Skalen Kontrolle $(d=0,64)$, Selbstvertrauen $(d=0,51)$, Vorwürfe $(d=0,62)$, Spannungen $(d=0,60)$ und Hilflosigkeit $(d=0,75)$ bewegen sich im mittleren und diejenigen für die Skalen Autonomie $(d=0,88)$ und Abhängigkeit $(d=$ 0,81 ) im hohen Bereich. Bemerkenswert ist, dass sich die Werte der Annäherungs- und der Vermeidungsskalen der IG vom Zeitpunkt t1 auf den Messzeitpunkt t2 verbessern. AuBer auf der Skala Anregung sind alle Veränderungen vom Messzeitpunkt t1 auf den Messzeitpunkt t2 signifikant.

Kovariate Geschlecht Da Frauen und Männer ungleich über die beiden Stichproben verteilt waren (Tab. 3), wurde entschieden, den Einfluss der Kovariate Geschlecht zu überprüfen. Überprüft wurden Einflüsse auf die Zeitpunkte insgesamt und einzeln sowie auf die Gruppe insgesamt und einzeln sowie auf die Gruppen * Zeit-Interaktionen. Es zeigten sich signifikante Gruppe * Zeit * Geschlecht-Effekte auf den Skalen Rollenklarheit in zwei Einzelvergleichen $\left(\mathrm{WG}_{\mathrm{t} 0} ; b=-0,158 ; p=0,041\right.$ und $\mathrm{WG}_{\mathrm{t} 2} ; b=-0,115 ;$ Wald $=$ 6,$845 ; p=0,009$ ) sowie auf die Skala Abhängigkeit auf die Gruppen insgesamt (Wald $=14,517 ; p=0,001)$. Signifikante Zeit * Geschlecht-Effekte waren für die Skala Autonomie insgesamt (Wald $=6,962 ; p=0,031$ ) und für den Einzelvergleich zum Zeitpunkt t1 ( $b=0,167$; Wald 4,329; $p=0,037$ ) für die Skala Spannungen festzustellen. Auf alle anderen Skalen hatte das Geschlecht keinen Einfluss. 
Tab. 6 Auswertung der Frage: Woran merken Sie im Alltag, dass Ihnen Coaching etwas gebracht hat?

\begin{tabular}{|c|c|c|c|}
\hline Kategorie & Ankerbeispiel (Kategorie, Nummer des Beispiels) & Anzahl & $\begin{array}{l}\text { Prozent } \\
(\%)\end{array}$ \\
\hline Umsetzung & „Ich habe direkt erste Massnahmen umgesetzt“ (U14) & 20 & 22,0 \\
\hline Zuversicht \& Hoffnung & $\begin{array}{l}\text { „Momentan fühle ich mich einfach gelöster und weiß, dass alles gut } \\
\text { wird.“ (ZH10) }\end{array}$ & 16 & 17,6 \\
\hline Bewusstheit (Person \& Umwelt) & ,... ich kenne meine und die Erwartungen der Beteiligten.“(B2) & 13 & 14,3 \\
\hline Planung & ,... ich weiss jetzt, was ich konkret machen muss ...“ (PL2) & 12 & 13,2 \\
\hline Zielorientierung & „Klarere Aussage zu meinem Ziel.“ (ZO1) & 11 & 12,1 \\
\hline Klarheit &,$\ldots .$. ich sehe sehr klar, und ... “ (K1) & 8 & 8,8 \\
\hline Perspektive & „,..., aber ich habe eine Vorstellung, wohin die Reise jetzt geht, ...“(P1) & 4 & 4,4 \\
\hline Selbstwert &,$\ldots$, sowie das Selbstwertgefühlt hat sich gesteigert.“(S2) & 3 & 3,3 \\
\hline Motivation & ..., und bin motiverter.“ (M3) & 3 & 3,3 \\
\hline Klärungsarbeit & $\begin{array}{l}\text { „Ich merke seither, dass es in mir arbeitet, und ich mir viele Gedanken } \\
\text { mache, was besprochen wurde.“ (K1) }\end{array}$ & 1 & 1,1 \\
\hline Total Nennungen & & 91 & 100 \\
\hline
\end{tabular}

\section{Qualitative Ergebnisse: Woran merken die Coachees im Alltag, dass sich etwas veränderte?}

Insgesamt 49 (79,0\%) Coachees nahmen zu dieser Frage Stellung. Gesucht wurde nach sprachlichen Äußerungen, die geeignet waren, Alltagswirkungen zu belegen. Aus 91 im Material gefundenen Textstellen wurden 10 Kategorien gebildet (Tab. 6). Die den Kategorien zugeordneten Äußerungen enthalten Hinweise, die als Handlungsfortschritte gedeutet werden können. Am meisten Nennungen wurden für die Kategorie Umsetzung (20;22,0\%) gefunden, gefolgt von Zuversicht und Hoffnung mit $16(16,8 \%)$ und Planung $(13 ; 17,6 \%)$. Am wenigsten Nennungen enthält die Kategorie Klärungsarbeit $(1 ; 1 \%)$.

\section{Diskussion}

Ziel dieser quasiexperimentellen Studie war die Evaluation der Wirksamkeit von Coachings basierend auf der Konzeption des SKETM. Im SKETM wird davon ausgegangen, dass Coaching den Coachees dabei hilft, Fortschritte in Richtung von selbstgesetzten Zielen zu erreichen. Dabei ist es nicht notwendig, dass das Anliegen während des Coachings vollständig bearbeitet werden muss. In der Regel ist es ausreichend, wenn die Coachees ihr Anliegen soweit bearbeiten, dass sie gut auf die selbständige Weiterbearbeitung des Themas vorbereitet sind. Ein erfolgreiches Coaching sollte sich in Veränderungen in der Zielannäherung, der Anliegenklärung und -umsetzung sowie in einem verbesserten Inkongruenzerleben spiegeln. Die Studie enthält Belege, dass dies gelungen ist. Das Ausmaß der gefundenen Effekte ist höher als die in den bisher vorliegenden Meta- analysen berichteten (Theeboom et al. 2014 ( $g^{1}=0,66$ für alle Studien; $g=0,39$ für Studien mit gemischten Designs); Jones et al. $2015(g=0,34)$; Sonesh et al. $2015(g=0,10))$. Da die Fragen der Skalen aus dem FEC-KU direkt auf das Anliegen Bezug nehmen, waren hier eher hohe Effekte zu erwarten. Aber auch die auf den Inkongruenz-Skalen gemessenen Effekte ( $d=0,34-0,88$ Interaktion; $d=0,44-0,98$ Zeit) bewegen sich am oberen Rand der Durchschnittseffekte aus den Metaanalysen. Die quantitativen Befunde werden durch die qualitativen bestätigt. In den freien Äußerungen zur Frage der Alltagswirkungen fanden sich Belege dafür, dass die Coachees in Hinsicht auf ihr Anliegen an Zuversicht gewinnen, die Umsetzung des Anliegens planen, erste Umsetzungsschritte ausführen, an Klarheit gewinnen und zielorientiert vorgehen.

Die deutlichsten Effekte waren bei beiden Gruppen auf der Zielannäherung zu verzeichnen. Dies stimmt mit der Befundlage in der Literatur überein (Grant 2003; Grant et al. 2009, 2010; Green et al. 2006; Gyllenstein und Palmer 2005). Bemerkenswert ist, dass die Werte der Interventionsgruppe auch einige Wochen nach dem Coaching auf dem gleichen Niveau verharren, was für eine nachhaltige Wirkung spricht. Die Großen Effektstärken von $d=1,52$ für die Interaktion und $d=1,51$ für den Zeiteffekt deuten zudem auf die praktische Bedeutsamkeit der Befunde.

Betrachtet man die Veränderungen auf den Skalen der Klärung und Umsetzung des Anliegens, zeigt sich, dass die Coachees Fortschritte wahrnehmen. Sie wissen genauer, was sie wollen, sie sehen Lösungen, fühlen sich in der Lage, die nächsten Schritte in Angriff zu nehmen, die mit dem Anliegen verbundene Befindlichkeit verbessert sich und sie nehmen eine erhöhte Rollenklarheit wahr. Sich seiner Ziele

\footnotetext{
${ }^{1}$ Hedges $g$ ist weitgehend mit Cohen's $d$ vergleichbar. Bei beiden wird
} eine Mittelwertdifferenz mit einer Standardabweichung verglichen. 
bewusst zu sein, diese als selbstkongruent wahrzunehmen und Wege zu kennen, die zu diesen Zielen führen, sind Grundelemente der Handlungskontrolle (Flammer 1990). Dass sich durch Coaching die Affektlage verbessern kann, zeigten schon verschiedene Studien (z. B. Finn et al. 2006; Green et al. 2006; Janßen et al. 2004; Offermanns 2003) und Rollenklarheit ist u. a. ein Prädiktor für Arbeitszufriedenheit und Arbeitsleistung (Kim et al. 2013). Diese Befunde beschränken sich zunächst auf das bearbeitete Anliegen. Trotzdem ist ein solches Ergebnis bemerkenswert, zeigt es doch, dass es durch Coaching gelingt, sehr unterschiedliche Anliegen zu bearbeiten und genau dort Nutzen zu stiften, wo er nachgefragt wird. Ob es einen Transfer-Effekt auf weitere Lebensbereiche gibt, ist hingegen nicht klar.

Inkongruenz Die Effekte auf das Inkongruenzerleben sind geringer. Dies war aber zu erwarten. Inkongruenz speist sich aus der gesamten Lebenssituation einer Person und nicht nur aus dem bearbeiteten Anliegen. Erzielt der Coachee bei seinem Anliegen Fortschritte, ist aber trotzdem zu erwarten, dass sich dies auch auf das Inkongruenzerleben auswirkt. Diese Vermutung wird von den Ergebnissen gestützt. Das Inkongruenzerleben verbessert sich vom ersten auf den zweiten Messzeitpunkt, aber nicht im gleichen Ausmaß wie die Anliegenklärung und -umsetzung. Entgegen der Erwartung und im Unterschied zu den Werten der Anliegenklärung und -umsetzung - diese bleiben vom zweiten auf den dritten Messzeitpunkt stabil - verbessern sich die Werte der Interventionsgruppe auf allen Inkongruenzskalen vom zweiten auf den dritten Messzeitpunkt. Dies könnte darauf hinweisen, dass die Resultate eines Coachings erst mit Zeitverzögerung auf weitere Lebensbereiche ausstrahlen.

\subsection{Grenzen der Studie}

Bei einem quasi-experimentellen Design können Selektionseffekte nie vollständig ausgeschlossen werden. Die Auswahl und die Zuteilung der Coachees zu den Gruppen erfolgten durch die Coaches und es konnte nicht kontrolliert werden, ob es hier zu verzerrenden Effekten kam. Die gesamte Stichprobe enthielt eindeutig mehr Männer als Frauen und die Frauen waren in der Interventionsgruppe übervertreten. Wie die Resultate zeigen, hatte das Geschlecht der Coachees einen Einfluss auf gewisse Skalen. Frauen wiesen auf der Skala Autonomie höhere (signifikant wurde nur der Effekt über alle drei Messzeitpunkte) und tiefere Werte auf der Skala Spannungen aus (Signifikante Effekte über alle Messzeitpunkte sowie für t0 und $\mathrm{t} 1$ einzeln; knapp signifikant für den Messzeitpunkt t2). Interaktionseffekte zeigten sich für die Skalen Rollenklarheit, Anerkennung und Abhängigkeit. Hier wiesen die Frauen der Wartekontrollgruppe im Vergleich zu den Männern erhöhte (Rollen- klarheit, Anerkennung) oder tiefere Werte aus (Abhängigkeit). Ob es sich hier einen systematischen Einfluss handelt und Frauen in gewissen Bereichen ein wenig mehr von Coaching profitieren, sich ihr Antwortverhalten unterscheidet oder ob es sich um einen Zufallseffekt handelte, kann auf der Basis der vorliegenden Daten nicht entschieden werden. Gegen weitere Selektionseffekte spricht, dass auf den Baseline-Variablen der Coachees, mit Ausnahme der Skala Anerkennung, keine Unterschiede gefunden wurden. $\mathrm{Ob}$ verzerrende Einflüsse auf die Resultate durch Coach-Charakteristika vorlagen, wurde, obwohl Daten zu Geschlecht, Alter und Erfahrung der Coaches vorlagen, nicht überprüft. Zum einen, weil hinsichtlich dieser Variablen keine Unterschiede zwischen den Gruppen vorlagen, zum anderen weil vor allem bei kleinen Stichproben durch das Hinzufügen von Kovariaten die Struktur der Fehlerkorrelationen und damit das Modell verändert werden kann und daher nicht zu empfehlen ist (Twisk 2013).

Obwohl die Stichprobe hinsichtlich Alter (Künzli 2009, 2012) der typischen Coaching-Klientel entspricht, können die Befunde dieser Untersuchung nicht generalisiert werden. Dazu ist die Stichprobe zu klein und der CoachingAnsatz zu spezifisch. Inwiefern die Stichprobe repräsentativ ist für Coachings, die nach den Vorgaben des SKETM durchgeführt werden, kann ebenfalls nicht beantwortet werden. Weder zu der Gesamtheit der Coaches, die nach dieser Methode arbeiten, noch zu deren Klienten und ihren Anliegen existieren Angaben, die entsprechende Schlüsse zulassen würden. Zum sozioökonomischen Status und zu Persönlichkeitsvariablen der Coachees wurden keine Daten erhoben. Eventuelle Einflüsse dieser Variablen konnten daher nicht geprüft werden.

Der Einfluss der sozialen Erwünschtheit die Ergebnisse konnte nicht geklärt werden. Die verwendeten Ergebnisvariablen beruhen auf Selbstberichten und die Coachees fühlten sich u. U. verpflichtet, ihren Coaches positive Rückmeldungen zu geben. Objektive Masse für einen möglichen Verhaltenstransfer konnten unter den gegebenen Umständen nicht erhoben werden. Dieser letzte Punkt verweist auf zukünftigen Forschungsbedarf. Zukünftige Studien sollten sich vermehrt mit objektivierbaren Transferwirkungen von Coaching befassen.

\section{Praktische und theoretische Implikationen}

Insgesamt scheint SKETM geeignet, für Coachees mit sehr verschiedenen Anliegen Nutzen zu erzeugen. SKETM orientiert sich an systemisch-konstruktivistischen Grundsätzen, ist hoch strukturiert (manualisiert), prozessorientiert und prozessdirektiv, bei gleichzeitiger inhaltlicher Abstinenz, findet an einem Tag statt und das Vorgehen ist gegenüber dem Coachee hoch transparent. Daraus lassen sich 
nun verschiedene für die Praxis und die Forschung relevante ableiten.

Im SKETM wird davon ausgegangen, dass die Effekte des Coachings im Wesentlichen auf die Elemente zurückzuführen sind. Der Versuch, einige dieser Elemente über die Behandlungstreue zu erfassen war nicht erfolgreich. Der Behandlungstreue-Index zeigte kaum Varianz und hatte daher keine Erklärungskraft. Die Untersuchung aller oder einzelner Elemente bleibt daher ein Forschungsdesiderat.

Zwar sind sich Lehrbuchautoren (z. B. Greif 2008; Lippmann 2013; Rauen 2008) weitgehend einig, dass ein Coachingprozess strukturiert, z.B. entlang von Phasen, innerhalb derer bestimmte Interventionen anzuwenden und/oder Arbeitsschritte abzuarbeiten sind, ablaufen sollte. Trotzdem dürfte der Strukturierungsgrad des SKETM aber einzigartig sein. Die Methode weist damit Ähnlichkeiten mit manualisierten Therapien wie z. B. der Kognitiven Verhaltenstherapie auf, deren Wirksamkeit in mehr als 250 Metaanalysen nachgewiesen ist (Hofmann et al. 2012). Ob und inwiefern dieser hohe Strukturierungsgrad bis hin zur Manualisierung für die Coachingpraxis einen Zusatznutzen bringt, ist aus Forschungssicht noch zu klären.

Ähnliches gilt für die Prozesstransparenz. Auch hier herrscht in der Literatur durchaus Konsens, dass Transparenz ein Qualitätsmerkmal ist (v. a. Schmidt 2013; aber auch Lippmann 2013 oder Rauen 2008). Dass hingegen der ganze Prozess und einzelne Teilschritte anhand eines Manuals erläutert werden, dürfte im Coaching eher die Ausnahme darstellen. Für die Praxis und die Forschung könnte es daher wertvoll sein, sich diesen Themen in Zukunft vermehrt zu widmen.

Für ein Coaching werden normalweise 4-6 Sitzungen mit einer Gesamtdauer von 8-10 h über einen Zeitraum von mehreren Wochen bis Monaten eingesetzt (Künzli 2009; Seiger und Künzli 2012; Stephan et al. 2010). Gewisse Autoren empfehlen sogar 8-10 Sitzungen (Berman und Bradt 2006). Als Argument für mehrere Sitzungen über einen längeren Zeitraum wird oft ins Feld geführt, dass der Coachee Zeit zur Reflektion und Umsetzung brauche (z. B. Lippmann 2013; Rauen 2005). Die Resultate dieser Studie lassen nun aber vermuten, dass Coaching auch sehr konzentriert, beschränkt auf einen Tag, eingesetzt werden kann und sich trotzdem erwünschte Effekte einstellen. Gestützt wird dieser Befund durch Burke und Linley (2007). Sie konnten positive Veränderungen der zielbezogenen Selbstkonkordanz und das Ziel-Commitment nach nur einer Coaching-Sitzung nachweisen. Zur Zielerreichung und zur Dauer der Sitzung machten die Autoren keine Angaben, was einen keinen direkten Vergleich zulässt.

SKETM setzt auf Prozessorientierung und -direktivität bei gleichzeitiger inhaltlicher Abstinenz. Im Coaching ist dies eine verbreitete Haltung, die in letzter Zeit aber kontrovers, v. a. unter dem Begriff Komplementärberatung, diskutiert wird (Böning und Fritschle 1997; Königswieser und Jochum 2006; Seewald und Schreyögg 2013; Fietze 2015). Der Begriff beschreibt Beratungen (Coachings), die in Abhängigkeit zum Klientenbedürfnis, zwischen inhaltlich-fachlicher und prozessorientierter Beratung wechseln. Welche Form(en) in Abhängigkeit vom Kontext optimal sind, ist aus Forschungssicht eine offene Frage.

$\mathrm{Zu}$ guter Letzt stellt sich die Frage, wie SKETM im Vergleich zu anderen Coaching-Ansätzen abschneidet.

\section{Ausblick}

Die Resultate dürfen als Hinweise für die Wirksamkeit von Coaching auf der Basis der Konzeption des SKETM gedeutet werden. Die Coachees nähern sich ihren Zielen an, ihre unmittelbar mit dem Anliegen verbundene Befindlichkeit verbessert sich, sie sehen ihre Ziele klarer, verfügen über eine Perspektive, die mit dem Anliegen verbundene Unsicherheit nimmt ab, sie gewinnen an Rollenklarheit und ihr Inkongruenzerleben vermindert sich. Halten wir uns noch einmal das Ziel von Coaching vor Augen: Coaching soll Menschen dabei helfen, zielorientiert und selbstkongruent zu handeln. Sieht man von den methodischen Mängeln der Studie ab, enthält sie einige Hinweise, dass genau dies gelungen ist.

Interessenkonflikt H. Künzli gibt an, dass kein Interessenkonflikt besteht.

Open Access Dieser Artikel wird unter der Creative Commons Namensnennung 4.0 International Lizenz (http://creativecommons.org/ licenses/by/4.0/deed.de) veröffentlicht, welche die Nutzung, Vervielfältigung, Bearbeitung, Verbreitung und Wiedergabe in jeglichem Medium und Format erlaubt, sofern Sie den/die ursprünglichen Autor(en) und die Quelle ordnungsgemäß nennen, einen Link zur Creative Commons Lizenz beifügen und angeben, ob Änderungen vorgenommen wurden.

\section{Literatur}

Barlow, D. H., Nock, M. K., \& Herson, M. (2009). Single case experimental designs. strategies for studying behavior change. Boston: Pearson.

Behrendt, P. (2004). Was kann Psychodrama zu einem erfolgreichen Coaching beitragen? - eine wirkfaktorenorientierte Prozessanalyse im Transfercoaching. Unveröffentlichte Diplomarbeit an der Universität Freiburg i.B.

Berman, H., \& Bradt, G. (2006). Different strokes for different folks. Professional psychology: research and practice. 37(3), 244-253. doi:10.1037/0735-7028.37.3.244.

Böning, U., \& Fritschle, B. (2005). Coaching fürs Business. Bonn: managerSeminare.

Böning, U., \& Fritschle, F. (1997). Veränderungsmanagement auf dem Prüfstand : Eine Zwischenbilanz aus der Unternehmenspraxis. Freiburg i.Br.: Haufe. 
Böning, U. \& Kegel, C. (2015). Ergebnisse der Coaching-Forschung. Aktuelle Studien - ausgewertet für die Coaching-Praxis. Heidelberg: Springer. doi:10.1007/978-3-662-43520-5.

Bozer, G., \& Sarros, J.C. (2012). Examining the effectiveness of executive coaching on coachees' performance in the Israeli context. International Journal Of Evidence Based Coaching \& Mentoring, $10(1), 14-32$

Burke, \& Linley (2007). Enhancing goal self-concordance through coaching. International Coaching Psychology Review, 2(1), $62-69$.

Cerni, T., Curtis, G.J., \& Colmar, S.H. (2010). Executive coaching can enhance transformational leadership. International Coaching Psychology Review, 5(1), 81-85.

Cohen (1988). Statistical power analysis for the behavioral sciences. Hillsdale: Lawrence Erlbaum.

Epstein, S., Pacini, R., Denes-Raj, V., \& Heier, H. (1996). Individual differences in intuitiv-experiential and analytical-rational thinking styles. Journal of Personality and Social Psychology, 71, $390-405$

Fietze, B. (2015). Coaching auf dem Weg zur Profession? Eine Professionssoziologische Einordnung. In A. Schreyögg \& C. SchmidtLellek (Hrsg.), Die Professionalisierung von Coaching (S. 3-21). Heidelberg: Springer.

Finn, F., Mason, C., \& Griffin, M. (2006). Investigating change over time - The effects of executive coaching on leaders' psychological states and behavior. 26th International Congress of Applied Psychology, Athens.

Flammer, A. (1990). Erfahrungen der eigenen Wirksamkeit. Bern: Huber.

Grant, A. M. (2003). The impact of life coaching on goal attainment, metacognition and mental health. Social Behavior and Personality, 31(3), 253-264.

Grant, A. M., \& Greene, J. (2001). Coach yourself: It's your life, what are you going to do with it? Make real changes in your life. London: Momentum.

Grant, A. M., Curtayne, L., \& Burton, G. (2009). Executive coaching enhances goal attainment, resilience and workplace well-being: a randomised controlled study. Journal of Positive Psychology, 4(5), 396-407.

Grant, A. M., Green, L.S., \& Rynsaardt, J. (2010). Developmental coaching for high school teachers: executive coaching goes to school. Consulting Psychology Journal: Practice and Research, 3, 151-168.

Grawe, K. (1998). Psychologische Therapie. Göttingen: Hogrefe.

Green, L. S., Grant, A. M., \& Rynsaardt, J. (2007). Evidence-based life coaching for senior high school students: building hardiness and hope. International Coaching Psychology Review, 2(1), 24-32.

Green, S., Oades, L.G., \& Grant, A.M. (2006). Cognitive-behavioral, solution-focused life coaching: enhancing goal striving, well-being and hope. The Journal of Positive Psychology, 1(3), 142-149. doi:10.1080/17439760600619849.

Greene, J., \& Grant, A. M. (2003). Solution focused coaching: managing people in a complex world. London: Momentum.

Greif, S. (2008). Coaching und ergebnisorientierte Selbstreflexion. Göttingen: Hogrefe.

Grosse-Holtforth, M., Grawe, K., \& Tamcan, Ö. (2004). Inkongruenzfragebogen INK. Göttingen: Hogrefe.

Gyllensten, K., \& Palmer, S. (2005). Can coaching reduce workplace stress: a quasi-experimental study. International Journal of Evidence Based Coaching and Mentoring, 3(2), 75-85.

Hofmann, S. G., Asnaani, A., Vonk, I. J. J., Sawyer, A. T., \& Fang, A. (2012). The efficacy of cognitive behavioral therapy. Cognitive Therapy and Research, 36(5), 427-440. doi:10.1007/s10608012-9476-1.

Huber, P. J. (1967). The behavior of maximum likelihood estimates under nonstandard conditions. Fifth Berkeley Symposium on Mathematical Statistics and Probability. Bd. 1, S. 221-233).
Jaenicke, C. (2009). Heilung als Ko-Kreation - Wechselseitige Regulierung im psychotherapeutischen Prozess. Psychotherapie Forum, 17(1), 9-14. doi:10.1007/s00729-009-0273-6.

Janßen, A., Mäthner, E., \& Bachmann, T. (2004). Erfolgreiches Coaching: Wirkfaktoren im Einzel-Coaching. Kröning: Asanger.

Jones, R. J., Woods, S.A., \& Guillaume, R.F. (2015). The effectiveness of workplace coaching: a meta analysis of learning and performance outcomes from coaching. Journal of Occupational and Organisational Psychology doi:10.1111/joop.12119.

Kim, S., Egan, T. M., Kim, W., \& Kim, J. (2013). The impact of managerial behavior on employee work-related reactions. Journal of Business and Psychology, 28(3), 315-330. doi:10.1007/s10869013-9286-9.

Kiresuk, T. J. \& Sherman, R. E. (1968). Goal attainment scaling: A general method for evaluating comprehensive community mental health programs. Community Mental Health Journal, 4(6), 443-453. doi:10.1007/BF01530764.

König, E., \& Volmer, G. (2012). Handbuch systemisches Coaching. Weinheim: Beltz.

Königswieser, R., \& Jochum, G. (2006). Das Zusammenspiel von Fach und Prozess-Know-How. Stuttgart: Klett-Cotta.

Kotte, S., Hinn, D., Oellerich, K., \& Möller, H. (2016). Der Stand der Coachingforschung: Kernergebnisse der vorliegenden Metaanalysen. Organisationsberatung, Supervision, Coaching, 23(1), 5-23. doi:10.1007/s11613-016-0444-6..

Kuhn, R. (2014). Das Glück der siebten Welle - Reflexionen zum Nutzen und Sinn in Beratungen. In I. Melter, E. Kanelutti-Chilas \& W. Stifter (Hrsg.), Zukunftsfeld Bildungs- und Berufsberatung (S. 33-56). Bielefeld: Bertelsmann.

Künzli, H. (2009). Wirksamkeitsforschung im Führungskräftecoaching. In E. Lippmann (Hrsg.), Coaching. Angewandte Psychologie für die Beratungspraxis (S. 280-294). Heidelberg: Springer.

Künzli, H. (2012). Wenn es nicht mehr passt. In R. Wegener, A. Fritze \& M. Loebbert (Hrsg.), Coaching entwickeln - Forschung und Praxis im Dialog (S. 107). Wiesbaden: Springer VS.

Künzli, H., \& Stulz, N. (2011). Individuumsorientierte Coaching-Forschung. In B. Birgmeier (Hrsg.), Coachingwissen (S. 159-169). Heidelberg: VS.

Künzli, H., \& Zihlmann, G. (2009). Wirkungen von Laufbahnberatungen erfassen. In D. Läge \& A. Hirschi (Hrsg.), Berufliche Übergänge. Psychologische Grundlagen der Berufs-Studien und Laufbahnberatung. Berlin: LIT.

Lai, Y.L., \& McDowall, A. (2014). A systematic review of coaching psychology: focusing on the attributes of effective coaching psychologists. International Coaching Psychology Review, 9(2), $120-136$

Leung, S. O. (2011). A comparison of psychometric properties and normality in 4-, 5-, 6-, and 11-point likert scales. Journal of Social Service Research, 37, 412-421. doi:10.1080/014883876.2011. 580697.

Lippmann, E. (2013). Coaching. Angewandte Psychologie für die Beratungspraxis. Heidelberg: Springer.

Loebbert, M. (2013). Professional Coaching. Konzepte, Instrumente, Anwendungsfelder. Stuttgart: Schäfer-Pöschel.

Meier, R. (2014). Systemisch-konstruktivistisches Einzel- und Teamcoaching im Management. Sternenfels: Wissenschaft \& Praxis.

Mayring, P. (2010). Qualitative Inhaltsanalyse: Grundlagen und Techniken. Weinheim: Beltz.

McKenna, D.D., \& Davis, S.L. (2009). Hidden in plain sight: the active ingredients of executive coaching. Industrial and Organizational Psychology, 2, 244-260. doi:10.1111/j.1754-9434.2009. 01143.x.

Meier, R. (2013). Theorie vom Selbstorganisierten Coaching. Sternenfels: Wissenschaft \& Praxis.

Möller, H., \& Kotte, S. (2011). Die Zukunft der Coachingforschung. Organisationsberatung, Supervision, Coaching, 18(4), 445-456. doi:10.1007/s11613-011-0256-7. 
Mummendey, H. D., \& Grau, I. (2008). Die Fragebogenmethode. Göttingen: Hogrefe.

Offermanns, M. (2003). Braucht Coaching einen Coach? Eine evaluative Pilotstudie. Stuttgart: ibidem.

Petzold, H. G., Schigl, B., Fischer, M., \& Höfner, C. (2003). Supervision auf dem Prüfstand. Wirksamkeit, Forschung, Anwendungsfelder, Innovation. Opladen: Leske + Budrich.

Radatz, S. (2003). Beratung ohne Ratschlag. Systemisches Coaching für Führungskräfte und Beraterinnen. Wien: Verlag systemisches Management.

Rauen, Ch. (2008). Coaching. Göttingen: Hogrefe.

Schlippe, A. von, \& Schweitzer, J. (2012). Lehrbuch systemische Therapie und Beratung. Göttingen: Vandenhoek \& Ruprecht.

Schmidt, G. (2013). Einführung in die hypnosystemische Therapie und Beratung. Heidelberg: Carl Auer.

Schreyögg, A. (2003). Coaching. Eine Einführung für Praxis und Ausbildung (6. Aufl.). Frankfurt: Campus.

Schreyögg, A. (2009). Die Wissensstruktur von Coaching. In B. Birgmeier (Hrsg.), Coachingwissen (S. 47-60). Wiesbaden: VS.

Schwarzer, R. (2001). Social-cognitive factors in changing health-related behavior. Current Directions in Psychological Science, 10, 47-51.

Schwarzer, R. (2004). Psychologie des Gesundheitsverhaltens. Einführung in die Gesundheitspsychologie. Göttingen: Hogrefe.

Seewald, C., \& Schreyögg, A. (2013). Coaching im Mittelstand. Organisationsberatung, Supervision, Coaching, 20, 243-244. doi:10. 1007/s11613-013-0337-x.

Seiger, C., \& Künzli, H. (2012). Schweizerischer Coachingmarkt aus Sicht der Coachs. Winterthur: Zürcher Hochschule für Angewandte Wissenschaften.
Sonesh, S.C., Coultas, C. W., Lacrenza, C.N., Marlow, S. L., Benishek, L.E., \& Salas, E. (2015). The power of coaching: a meta analytic investigation. Coaching, 8(2), 73-95.

Spence, G. B., \& Grant, A. (2007). Professional and peer life coaching and the enhancement of goal striving and well-being: an exploratory study. The Journal of Positive Psychology, 2, 185-194.

Steinmetz, B. (2005). Stressmanagement für Führungskräfte. Hamburg: Kovac.

Stephen, M., Gross, P.P., \& Hildebrandt, N. (2010). Management von Coaching. Stuttgart: Kohlhammer.

Theeboom, T., Beersma, B., \& Vianen, A.E. van. (2014). Does coaching work? A meta-analysis on the effects of coaching on individual level outcomes in an organizational context. The Journal of Positive Psychology, 9(1), 1-18. doi:10.1080/17439760.2013. 837499.

Twisk, J. W. R. (2013). Applied longitudinal data analyses for epidmiology. A practical guide. Cambridge: Cambridge University Press.

Watson, D., Clark, L. A., \& Tellegen, A. (1988). Development and validation of brief measures of positive and negative affect: the PANAS Scales. Journal of Personality and Social Psychology, 54, 1063-1070.

White, H. (1980). A heteroskedasticity-consistent covariance matrix estimator and a direct test for Heteroskedasticity. Econometrica, 48, 817-838.

Zeeger, S.L., Liang, K.-Y., \& Albert, P. S. (1988). Models for longitudinal data: A generalized estimating equation approach. Biometrics, 44, 1049-1060. 